\title{
Using Optical Sensor Nanoparticles for Imaging Chemical Dynamics is Marine Systems
}

\author{
Klaus. Koren ${ }^{1}$, Maria Mosshammer ${ }^{2}$,Kasper E. Brodersen ${ }^{2}$, Erik C. Trampe ${ }^{2}$, Michael Kühl, ${ }^{2,3}$ \\ ${ }^{1}$ Department of Bioscience - Microbiology, Aarhus University, Ny Munkegade 116, 8000 Aarhus, \\ Denmark, \\ ${ }^{2}$ Marine Biological Section, University of Copenhagen, Strandpromenaden 5, 3000Helsingør, \\ Denmark \\ ${ }^{3}$ Climate Change Cluster, University of Technology Sydney, Sydney, Australia \\ klaus.koren@bios.au.dk
}

\begin{abstract}
:
Dynamic chemical gradients are prevalent in many surface-associated marine habitats due to limited diffusion and high volume specific reaction rates of key analytes such as $\mathrm{O}_{2}$. For example, photosynthetic $\mathrm{O}_{2}$ production in biofilms and algal tissues can change $\mathrm{O}_{2}$ levels within a few minutes in response to changes in irradiance. In addition, many marine systems (e.g. corals or biofilms) are structurally complex and heterogeneous. We will discuss different possibilities of using optical nanosensors to visualize chemical dynamics in such heterogeneous systems. Marine applications of optical nanosensors in combination with different imaging techniques will be presented. By using optical nanosensor-based chemical imaging, it is possible to study structurally complex samples at high resolution; a clear advantage to other existing sensor approaches.
\end{abstract}

Key words: Optical sensors, nanoparticles, chemical imaging, luminescence, marine applications.

\section{Introduction}

Marine environments are highly active ecosystems responsible for a major part of global primary production, and essential for biogeochemical cycling of carbon and essential nutrients. Within the marine environment several interesting hotspots and species can be found. Symbiont bearing corals for example show very high photosynthetic activity in the light and respiration in the dark [1,2]. Also marine aquatic plants like seagrasses manage to thrive in anoxic and toxic $\mathrm{H}_{2} \mathrm{~S}$ containing sediments [3]. In order to understand the chemical microenvironments involved within such marine species or environments, it is important to be able to measure, or even better, visualize them. Although sensor technology is highly used within marine sciences most of the commonly used sensor solutions have difficulties resolving such structurally complex systems in 2D.

Here we describe the use of optical nanosensors as a new method to visualize chemical distributions and dynamics on complex samples.

\begin{abstract}
Materials and Methods
We used optical nanosensors for $\mathrm{O}_{2}$ and $\mathrm{pH}$, as prepared by a simple and effective precipitation method [4]. In brief, a mixture of a polymer, an analyte sensitive dye and a reference dye are dissolved in THF and rapidly poured into an at least 10 times larger, vigorously stirred volume of water. After THF removal and concentration at elevated temperature, the particle suspension is ready to be used. Imaging of the analyte sensitive emission is performed using either a ratiometric approach using a standard SLR camera or by lifetime based imaging.
\end{abstract}

\section{Results and discussion}

Over the last years, we have shown that optical nanosensors are valuable tools for studying complex chemical landscapes and dynamics in different marine systems. Nanosensors can e.g. be used to map the surface $\mathrm{O}_{2}$ levels on a coral fragment by spray-painting the fragment and subsequent imaging (see Fig.1) [5]. Also chemical processes within the rhizosphere of seagrasses can be investigated at great detail 
using optical nanosensors embedded in an artificial sediment $[6,7]$.

We were also able to study biofilms growing on rock surfaces (beach rock) using this methodology. Here again a special way of applying the nanosensors was used. The complex surfaces were coated (painted) with a sensor layer and subsequently imaged (manuscript in preparation). As the decribed nanosensors can be enhanced in functionality, for example by making them magnetic, the list of potential applications is endless. We have successfully collected and held in place magnetically functionalized nanosensors on a seagrass leave surface with the help of a magnetic field (manuscript in preparation). This enables imaging even at high flow speeds.

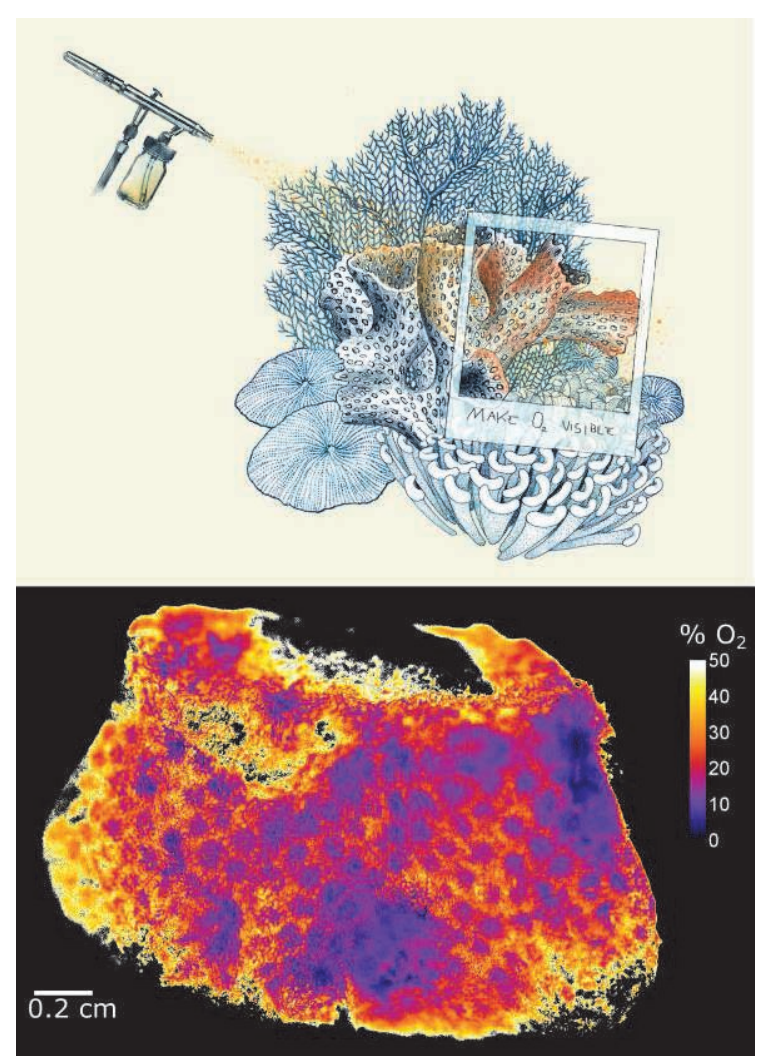

Fig. 1. By combining optical nanosensors and imaging techniques it is possible to visualize the complex $\mathrm{O}_{2}$ distribution on the surface of a coral fragment. The coral is stray-painted with optical nanosensors and later imaged using a ratiometric imaging approach.

Imaging of the analyte-dependent sensor luminescence can either be performed by applying a ratiometric approach or a lifetimebased approach. In a ratiometric system the emission of an analyte sensitive dye and an insensitive dye are compared in order to compensate for e.g. fluctuations in nanosensor density and uneven illumination of the monitored sample. When using lifetime-based imaging the lifetime of the analyte sensitive dye is measured directly. Both methods have advantages and shortcomings. Sample constrains (e.g. background fluorescence) determine which methods is preferable.

Compared to other possible sensor techniques, nanosensors can have clear advantages. Microsensors enable only point measurements, while imaging using nanosensors enables 2D (and even 3D) visualization. Planar sensor films (opt(r)odes) can be used to realize 2D imaging as well, but are limited to less structurally complex samples as the film needs to be in physical contact with the sample.

\section{Conclusion}

Optical nanosensors present new possibilities for studying chemical distributions and dynamics in complex samples. The flexibility in terms of applying the nanosensors enables imaging of structurally challenging samples like uneven and porous samples.

\section{References}

[1] K.E. Brodersen, M. Lichtenberg, P.J. Ralph, M. Kühl, D. Wangpraseurt, J. R. Soc. Interface. 11 (2014) 20130997. doi:10.1098/rsif.2013.0997.

[2] M. Kühl, Y. Cohen, T. Dalsgaard, B.B. Jørgensen, N.P. Revsbech, Mar. Ecol. Prog. Ser. 117 (1995) 159-172.

[3] J. Borum, O. Pedersen, T.M. Greve, T.A. Frankovich, J.C. Zieman, J.W. Fourqurean, C.J. Madden, J. Ecol. 93 (2005) 148-158. doi:10.1111/j.1365-2745.2004.00943.x.

[4] G. Mistlberger, K. Koren, E. Scheucher, D. Aigner, S.M. Borisov, A. Zankel, P. Pölt, I. Klimant, Adv. Funct. Mater. 20 (2010) 18421851. doi:10.1002/adfm.201000321.

[5] K. Koren, S.L. Jakobsen, M. Kühl, Sensors Actuators B Chem. (2016) 19-21. doi:10.1016/j.snb.2016.05.147.

[6] K. Koren, K.E. Brodersen, S.L. Jakobsen, M. Kühl, Environ. Sci. Technol. 49 (2015) 22862292. doi:10.1021/es505734b.

[7] K. Elgetti Brodersen, K. Koren, M. Lichtenberg, M. Kühl, Plant. Cell Environ. 39 (2016) 16191630. doi:10.1111/pce.12740. 